\title{
IMPORTANCIA DE LA FLORA ORNAMENTAL EN LA AEROPALINOLOGIA DE SEVILLA (ESPAÑA)
}

\author{
Francisco José GONZÁLEZ MINERO, Pilar CANDAU y M. Luis GONZÁLEZ ROMANO
}

\begin{abstract}
RESUMEN. Importancia de la flora ornamental en la aeropalinología de Sevilla (España). El trabajo se ha realizado con un captador Cour que muestreó el aire de la ciudad durante ocho años consecutivos (19871994). En total se han establecido 32 tipos polínicos ornamentales, pertenecientes a 30 familias diferentes y que suponen el $30 \%$ del polen total recogido. Los tipos con una representación al superior al $0.2 \%$ son, por orden de importancia: Cupressaceae, Platanus, Palmae, Moraceae, Fraxinus, Salicaceae, Casuarina, Acer, Ulmus y Citrus. La mayoría de los tipos restantes son de carácter entomófilo, por lo que su aparición en el aire es esporádica y en concentraciones inferiores a 1 grano semanal $/ \mathrm{m} 3$. El polen ornamental supone un alto porcentaje de polen total entre diciembre y abril. La mayoría de los tipos aparecen en la primera mitad del año (excepto Palmae y Casuarina), produciéndose las semanas de máxima recogida de polen antes de la semana quince del año. La variación, a lo largo del año, de las concentraciones polínicas semanales se caracterizan por un perfil gráfico homogéneo, excepto Palmae y Citrus, en los que se observan continuas subidas y bajadas de las concentraciones.
\end{abstract}

Palabràs clave: Aeropalinología, flora ornamental, polen, Sevilla.

ABSTRACT. Importance of the ornamental flora in the aeropalynology of Seville (Spain). A Cour trap was used to sample the air of the city for eight consecutive years (1987-1994). A total of 32 pollen types has been established, belonging to 30 different families, and making up $30 \%$ of the total pollen collected. The types with a representation higher than $0.2 \%$ are, in order of importance: Cupressaceae, Platanus, Palmae, Moraceae, Fraxinus, Salicaceae, Casuarina, Acer, Ulmus and Citrus. Most of the remaining types are entomophilous, so that their appearance in the air is sporadic and at concentrations lower than 1 grain weekly/m3. The ornamental pollen comprises a high percentage of the total pollen between December and April. Most of the types appear in the first half of the year (except Palmae and Casuarina), with the weeks of maximum pollen collection being before week fifteen of the year. The variation in the weekly pollen concentrations throughout the year shows a homogeneous graphic profile, except Palmae and Citrus, which have continual rises and falls in concentration.

Key words. Aeropalynolgy, ornamental flora, pollen, Seville.

\section{INTRODUCCIÓN}

Dado el interés creciente que despierta el polen de procedencia ornamental en aeropalinología (Donini \& Sutra, 1987; Caramiello et al., 1992; Díaz de la Guardia y
Blanca, 1994; Trigo y Fernández, 1995), se ha realizado un estudio aeropalinológico del mismo llevado a cabo en la ciudad de Sevilla, aunque, con anterioridad, se publicaron resultados parciales de la misma ciudad sobre la aeropalinología de Citrus y Platanus hybrida 
(Cepeda y Candau, 1990).

La ciudad de Sevilla está situada en el valle bajo del Río Guadalquivir a orillas de éste, las coordenadas geográficas son $37^{\circ} 23^{\prime} \mathrm{N}$ y $5^{\circ} 58^{\circ} \mathrm{W}$. La altitud de la ciudad oscila entre 0 y 28 metros. La ciudad posee un clima mediterráneo moderado caracterizado por inviernos suaves y veranos prolongados y calurosos. La temperatura media anual es de $18.1^{\circ} \mathrm{C}$, la media anual de las temperaturas máximas es de $24^{\circ} \mathrm{C}$ y la media de las mínimas de $11^{\circ} \mathrm{C}$. Los meses más cálidos son julio y agosto $\left(26.5\right.$ y $26.2^{\circ} \mathrm{C}$ de temperatura media respectivamente) y los más fríos diciembre y enero (11 y $10.7^{\circ} \mathrm{C}$ respectivamente). La precipitación anual media es de $606.2 \mathrm{~mm}$, estando irregularmente repartida a lo largo del año y a lo largo de los años. Las horas de sol anuales son 2877 y la humedad relativa media del 68\% (datos medios del periodo 1922-1994, medidos en el observatorio de Tablada).

La larga historia de la ciudad, que ha sido ocupada sucesivamente por las distintas civilizaciones que han colonizado la Península Ibérica, le han dejado como legado una flora ornamental rica y diversificada, con especies procedentes de todos los continentes que han encontrado en su clima favorable unas condiciones óptimas para su desarrollo. Como resultado de todas estas circunstancias, la relación de plantas ornamentales que potencialmente pueden estar presentes en el espectro polínico de la ciudad, está compuesta por 800 especies diferentes pertenecientes a 47 familias (Elías Bonells, 1983; Andrés Camacho, 1991), repartidas en calles y avenidas, 6 grandes espacios verdes y 18 pequeños jardines. Las familias mejor representadas son: Cupressaceae, Lauraceae, Moraceae, Ulmaceae, Salicaceae, Platanaceae, Fabaceae, Punicaceae, Anacardiaceae, Simaroubaceae, Meliaceae, Rutaceae, Araliaceae, Apocynaceae, Solanaceae, Verbenaceae, Oleaceae, Caprifoliaceae y Palmae.
Los taxa mejor representados en el sector urbano en el que se encuentra el captador de polen son: Platanus hispanica, Fraxinus ornus, Ulmus minor, Cupressus sempervirens, Acer negundo, Casuarina cunninghamiana, Salix alba, Populus alba, Phoenix dactylifera, Washingtonia filifera, Ligustrum vulgare, Citrus auriantium, Brachychiton populneum, Melia azedarach, Robinia pseudoacacia, Tipuana speciosa y Jacaranda ovalifolia.

\section{MATERIAL Y MÉTODOS}

Se han realizado muestreos semanales del aire de Sevilla durante los años 1987-1994 empleando un captador Cour situado a 15 de metros de altura en la azotea de la Facultad de Farmacia. La metodología utilizada en el procesado de las muestras ha sido la descrita por Cour en 1974.

Para la identificación de los tipos polínicos se han consultado los siguientes trabajos bibliográficos: Gálvez y Ubera (1985), Valdés et al. (1987) y González Romano y Candau (1990, 1994). Así mismo se ha utilizado la palinoteca de polen ornamental del Departamento de Biología Vegetal y Ecología de Sevilla (Facultad de Farmacia).

Para los tipos polínicos cuya representación en el espectro es igual o superior al $0.2 \%$ del polen total recogido, se ha calculado la semana de máxima recogida polínica (SMRP) y el periodo de polinización principal (PPP). El periodo de polinización principal se define como aquellas semanas del año entre las que se recoge el 5 y el $95 \%$ del polen anual de un determinado tipo (Nilsson y Persson, 1981).

Se han construido las curvas polínicas anuales de los tipos polínicos más representativos, en la que se muestran la variación, a lo largo del año, de las concentraciones polínicas semanales, representando en el eje de abcisas las semanas y meses, y en el de ordenadas las 


\begin{tabular}{|c|c|c|c|}
\hline Tipo & Familia & Taxones que incluye & Atación \\
\hline Acacia & Leguminosae & $\begin{array}{l}\text { Acacia baileyana, A. dealbata, A. decurrens } \\
\text { A. farnesiana, A. karoo, A. melanoxylon, } \\
\text { A. retinoides, A. saligna }\end{array}$ & 0.11 \\
\hline Acer & Aceraceae & Acer negundo, A. pseudoplatanus & 0.34 \\
\hline Ailanthus & Simaroubaceae & Ailanthus altissima & $<0.01$ \\
\hline Brachychiton & Sterculiaceae & Brachychiton acerifolia. B. populneum & 0.05 \\
\hline Casuarina & Casuarinaceae & C. cunninghamiana, C. stricta & 0.36 \\
\hline Citrus & Rutaceae & $\begin{array}{l}\text { Citrus auriantium, C. bergamia, } C \text {. deliciosa, } \\
\text { C. limon, C. myrtifolia, C. sinensis }\end{array}$ & 0.24 \\
\hline Cocculus & Menispermaceae & Cocculus laurifolius & 0.07 \\
\hline Cupressaceae & Cupressaceae & $\begin{array}{l}\text { Cupressus sempervirens, } C \text {. arizonica, C. macrocarpa, } \\
\text { Chamaecyparis lawsoniana, } \\
\text { Thuja plicata, Platycladus orientalis }\end{array}$ & 11.89 \\
\hline Elaeagnus & Eleagnaceae & Elaeagnus angustifolia, E. umbellata & $<0.01$ \\
\hline Fraxinus & Oleaceae & Fraxinus angustifolia, F. excelsior, F. ornus & 1.18 \\
\hline Hedera & Araliaceae & Hedera helix & $<0.01$ \\
\hline Ilex & Aquifoliaceae & Ilex aquifolium & $<0.01$ \\
\hline Juglans & Juglandaceae & Juglans regia & 0.02 \\
\hline Lantana & Verbenaceae & Lantana camara, L. sellowiana & $<0.01$ \\
\hline Ligustrum & Oleaceae & Ligustrum japonicum, L. lucidum, L. vulgare & 0.04 \\
\hline Magnolia & Magnoliaceae & Magnolia grandiflora & $<0.01$ \\
\hline Melia & Meliaceae & Melia azedarach & $<0.01$ \\
\hline Moraceae & Moraceae & Morus nigra, M. alba, Broussonetia papyrifera & 1.51 \\
\hline Myoporum & Myoporaceae & Myoporum tenuifolium & $<0.01$ \\
\hline Nerium & Apocynaceae & Nerium oleander & $<0.01$ \\
\hline Palmae & Palmae & $\begin{array}{l}\text { Phoenix sp., Trachycarpus fortunei } \\
\text { Washingtonia sp. }\end{array}$ & 2.23 \\
\hline Phytolacca & Phytolaccaceae & Phytolacca dioica & $<0.01$ \\
\hline Platanus & Platanaceae & Platanus hispanica & 11.05 \\
\hline Rosaceae & Rosaceae & Crataegus sp., Pyrus sp., Rosa sp. & 0.02 \\
\hline Salicaceae & Salicaceae & Salix sp., Populus sp. & 0.38 \\
\hline Sambucus & Caprifoliaceae & Sambucus nigra & $<0.01$ \\
\hline Schinus & Anacardiaceae & Schinus molle & $<0.01$ \\
\hline Tamus & Dioscoridaceae & Tamus communis & $<0.01$ \\
\hline Tilia & Tiliaceae & Tilia platyphyllos & 0.01 \\
\hline Ulmus & Ulmaceae & Ulmus minor, $U$. glabra & 0.29 \\
\hline Viburnum & Caprifoliaceae & $\begin{array}{l}\text { Viburnum tinus, V. opulus, V. suspensum } \\
\text { V.odoratissimun, V. rhytidophyllum }\end{array}$ & 0.11 \\
\hline Vitex & Verbenaceae & Vitex agnus-castus & $<0.01$ \\
\hline
\end{tabular}

Tabla 1. Tipos polínicos identificados y porcentajes de representación de los mismos en el polen total recogido. Valores medios de los ocho años de muestreo. Pollen types identified and their percentage of representation in the total pollen collected. Values are means of the eight years of sampling.

concentraciones en granos/m3 (al ser muy variable las concentraciones alcanzadas por los distintos taxa, se usa la escala más apropiada para propiciar en cada caso una visualización adecuada).

\section{RESULTADOS}

En total, se han identificado 32 tipos polínicos diferentes procedentes de la flora ornamental, que corresponden a 30 familias, y 


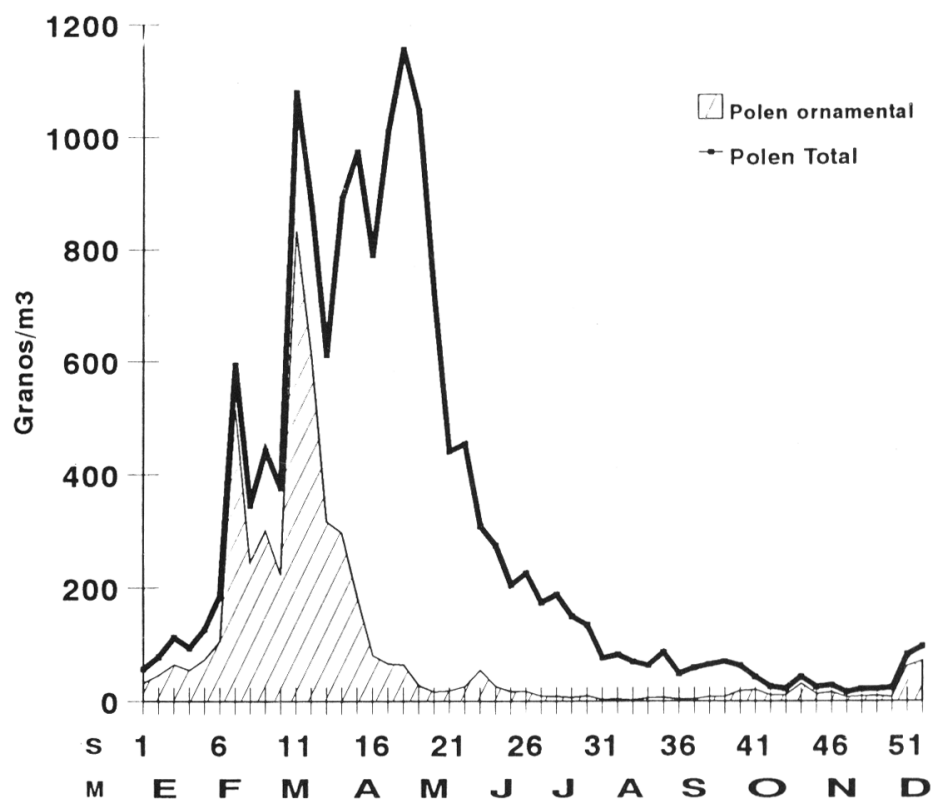

Figura 1. Variación de las concentraciones polínicas semanales del polen ornamental globalmente considerado y del polen total. Valores medios de los ocho años de muestreo. Variation in the weekly pollen concentrations of ornamental pollen considered overall, and of total pollen. Values are means of the eight years of sampling.

representan aproximadamente el $30 \%$ del polen total recogido (tab. 1). Los tipos más importantes, con un porcentaje de representación en el polen total recogido superior al $0.20 \%$ son: Cupressaceae $(11.89 \%)$, Platanus (11.05\%), Palmae (2.23\%), Moraceae (1.51\%), Fraxinus (1.18\%), Salicaceae $(0.38 \%)$, Casuarina $(0.36 \%)$, Acer $(0.34 \%)$, Ulmus $(0.29 \%)$ y Citrus $(0.24 \%)$. Los tipos restantes, se dividen en aquellos que poseen una una representación igual o superior al $0.01 \%$ e inferior al $0.2 \%$ (Acacia, Brachychiton, Cocculus, Juglans, Ligustrum, Rosaceae y Tilia), y en los que su representación es inferior al 0.01\% (Ailanthus, Elaeagnus, Hedera, Ilex, Lantana, Magnolia, Melia, Myoporum, Melia, Phytolacca, Sambucus, Schinus, Tamus, Viburnum y Vitex). Estos dos grupos se caracterizan por una presencia en el aire esporádica y discontinua, en concentraciones que generalmente no superan 1 grano semanal/ m3.

La dinámica atmosférica del polen ornamental globalmente considerado, se caracteriza por una presencia continua durante todas las semanas del año, constituyendo una parte importante del polen total recogido entre diciembre y abril, intervalo de tiempo en el que las curvas del polen ornamental y polen total se desarrollan de una forma paralela (Fig. 1). Esta participación en el polen total recogido es especialmente remarcable en las semanas 7 (en la que se produce la máxima recogida polínica de Cupressaceae) y 11 (coincidiendo con la semana de máxima recogida polínica de Platanus) (fig. 2).

Las curvas polínicas de los diez tipos cuantitativamente más importantes, se caracterizan por su homogeneidad, con uno o dos picos de máxima concentración. Esto no es válido para Palmae y Citrus, en los que se aprecian un perfil irregular con constantes subidas y bajadas (fig. 2). En esta figura también se observa que los niveles de concentración atmosférica varían significativamente entre los distintos tipos: en 

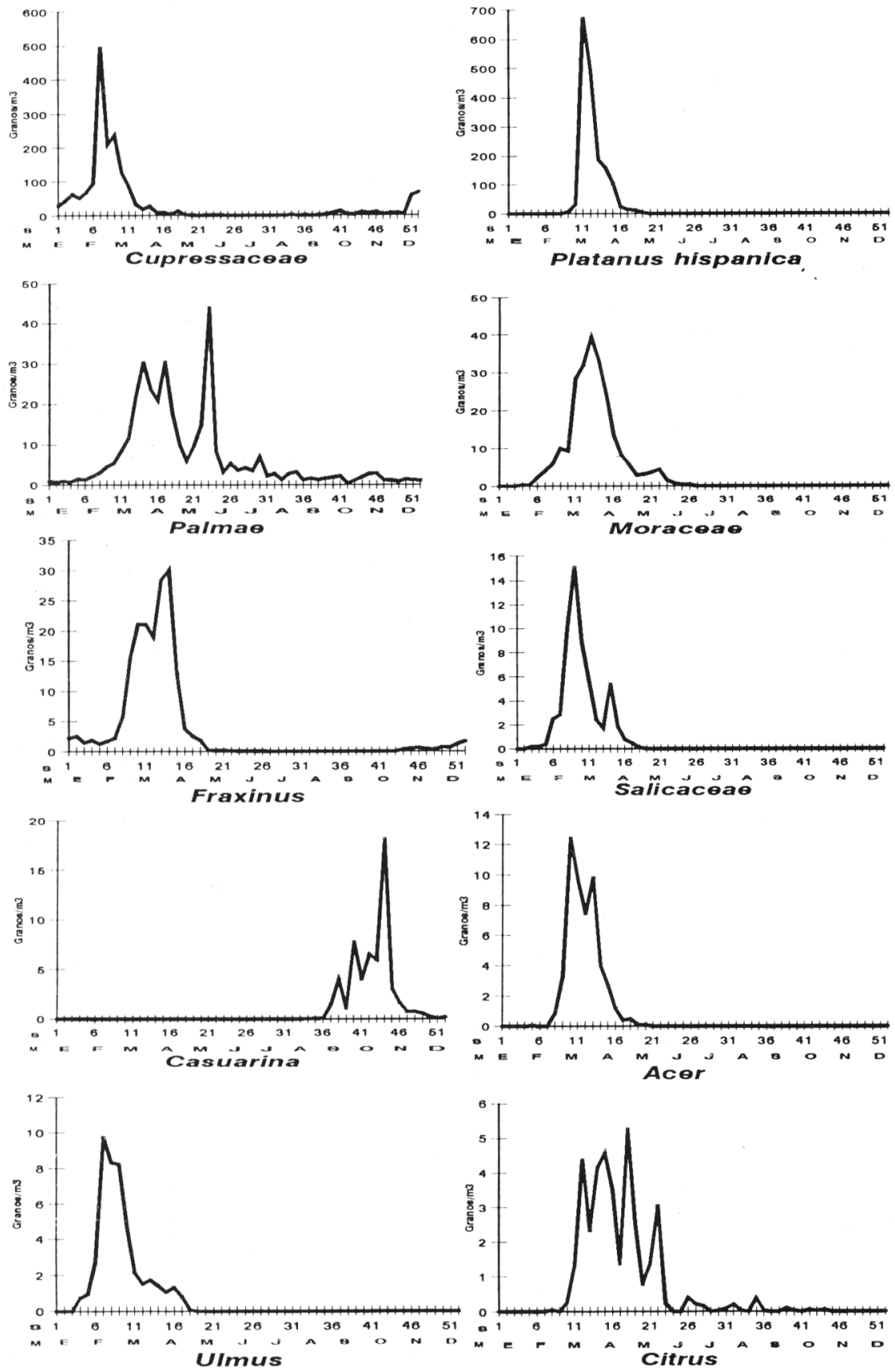

Figura 2. Variación de las concentraciones polínicas semanales de los diez tipos de origen ornamental cuantitativamente más abundantes. Valores medios de los ocho años de muestreo. Variation in the weekly pollen concentrations of the ten most abundant types of ornamental origin. Values are means of the eight years of sampling. 


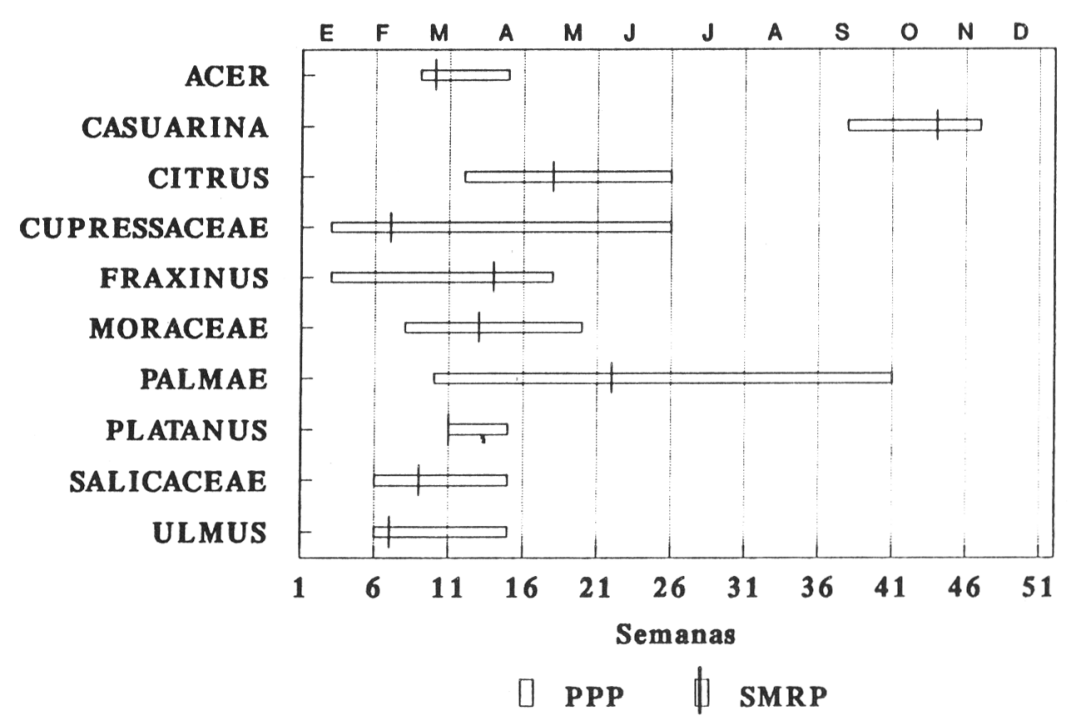

Figura 3. Periodos de polinización principal y semanas de máxima recogida polínica de los tipos con representación superior al $0.2 \%$. Valores medios de los ocho años de muestreo. Periods of main pollination and weeks of maximum pollen collection for the types with representation higher than $0.2 \%$. Values are means of the eight years of sampling.

Platanus se registran 500-700 granos/m3 en marzo; en Cupressaceae se miden 100-500 granos $/ \mathrm{m} 3$ en febrero y marzo; en Palmae (abril y junio), Moraceae (marzo y abril) y Fraxinus (marzo y abril) se rebasan los 20 granos/m3; en Salicaceae (febrero), Casuarina (noviembre) y Acer (marzo) se sobrepasan los 10 granos/ m3; y en Ulmus y Citrus nunca se superan los 10 granos/m3. La información aeropalinológica recogida en la figura 2 , se complementa con la representación de los periodos de polinización principal (PPP) y las semanas de máxima recogida de polen (SMRP) (fig. 3). Analizando esta última figura se constata que Cupressaceae y Fraxinus son los tipos polínicos más tempranos (el periodo comienza en la tercera semana de enero) y Casuarina el más tardío (final de septiembre); también se aprecia que en todos los tipos, excepto Palmae y Cupressaceae, la duración del periodo de polinización principal es inferior a 15 semanas, produciéndose en la mayoría de los taxa en la primera mitad del año. Por último cabe destacar que las semanas de mayor recogida de polen se registran antes de la semana quince del año, excepto en Palmae y Casuarina.

\section{DISCUSIÓN}

La alta densidad, tanto cualitativa como cuantitativa, que la flora ornamental tiene en Sevilla, hace que en el aire de la ciudad se hayan identificado 32 tipos polínicos diferentes de procedencia ornamental y que éstos supongan el $30 \%$ del polen total recogido (Tab. 1). Este hecho aerobiológico es una de las características del espectro polínico de Sevilla, y es interesante compararlo con los resultados publicados de otras ciudades andaluzas: en Cádiz y Huelva, el polen de procedencia ornamental no supera el $10 \%$ del polen total recogido (Candau y González Minero, 1992; González Minero y Candau; 1995), mientras que en Málaga este porcentaje no llega al 20\% (Recio et al., 1995). 
Los tipos polínicos identificados corresponden a plantas más abundantes en el espacio urbano con respecto al medio natural no urbano, de ahí que no se considere el polen de Myrtaceae, Olea europaea, Pinaceae y Quercus, ya que si bien son plantas que se encuentran en la flora ornamental de Sevilla, su representación en los espacios verdes de la ciudad es muy pequeña. Por otra parte, sería muy dificil explicar que los pocos eucaliptos, olivos, pinos y encinas dispersos en parques y avenidas de Sevilla, son los productores exclusivos de las altas concentraciones que habitualmente se detectan de sus respectivos tipos polínicos (González Romano et al., 1992, 1993). En este punto conviene matizar que en el espectro polínico de una determinada localidad aparecen reflejados los elementos de la vegetación autóctona y alóctona distribuidos en un radio de 50-100 kms. (Cambon, 1981), según este argumento no es posible precisar la distancia que ha recorrido un tipo polínico en su desplazamiento desde la planta hasta el captador. En cambio, sí se aceptan estimaciones en los casos de palinomorfos marcadores, es decir polen de especies que no están presentes en la vegetación que potencialmente está bajo la influencia del captador, y cuya presencia en el aire denota un necesario transporte desde largas distancias, tal es el caso de Castanea sativa en Huelva (González Minero y Candau, 1995) y Cannabis sativa en Málaga (Recio et al., 1995).

Si se consultan los trabajos de Trigo y García (1990), Trigo et al. (1994) y Trigo y Fernández $(1994,1995)$ es posible desdoblar algunos de los tipos aparecidos en la tab. 1 en los que, bajo una amplia denominación taxonómica, se agrupa polen con gran similitud morfológica: Acacia, Brachychiton, Palmae y Salicaceae. Esta posibilidad no se ha considerado en estos tipos, ya que, por regla general, o bien aparecen en bajas concentraciones atmosféricas, o bien su significación alergológica descrita hasta el momento es baja. Por lo que su estudio aeropalinológico pormenorizado requiere gran cantidad de tiempo y a veces, el empleo del microscopio electrónico, condicionantes que en la mayoría de los casos, se oponen a uno de los axiomas principales de la aerobiología: encontrar un equlibrio entre la identificación eficaz de los tipos importantes en cada momento, con el tiempo empleado en dicha identificación (Cambon, 1981). No osbtante, siempre existe la posibilidad de realizar estudios aeropalinológicos más profundos (tanto prospectivos como retrospectivos) de aquellos tipos polínicos sobre los que se haya despertado un determinado interés.

La composición cuantitativa del espectro polínico de cualquier localidad está sujeta a los efectos de sobrerrepresentación ejercida por aquellas plantas más cercanas al captador, así se deriva del trabajo de González Romano et al. (1993), en el que se muestra que la composición del espectro polínico de Sevilla varía en función de la ubicación del captador; esta premisa se puede ejemplarizar en nuestro caso con Platanus hispanica, el segundo tipo más importante identificado en el aire (tab. 1) y del que se han registrado las concentraciones polínicas más elevadas (fig. 2), situación en parte generada por la presencia de numerosos ejemplares de plátanos de sombra en las cercanías del captador, y que posiblemente no se repetiría si éste estuviera situado en otra parte de la ciudad. Otros aspectos destacables de la aeropalinología ornamental de Sevilla, es el alto porcentaje de polen de Palmae y la presencia en cantidades apreciables de Citrus, a pesar de su carácter entomófilo (tab. 1), ambos casos consecuencia de la abundancia de estas palntas en la ciudad. Finalmente, la identificación, en pequeñas concentraciones, de un largo número de tipos entomófilos (tab. 1) enriquecen el espectro desde un punto de vista taxonómico y son una prueba de la riqueza florística de la flora ornamental de Sevilla.

La variación, a lo largo del año, de las 
concentraciones polínicas semanales de los tipos polínicos más importantes no difiere substancialmente de lo ya descrito en otras ciudades andaluzas (Ruiz de Clavijo et al., 1988; Fernández et al., 1990; Candau y González Minero, 1992; Cabezudo et al., 1994; González Minero y Candau, 1995). Dichos patrones aeropalinológicos son, por regla general, homogéneos y corresponden a plantas con floración precoz de las que se recogen las máximas cantidades de polen en las primeras semanas del año. Esta circunstancia debe ser tenida en cuenta a la hora de explicar los casos de alergia en los primeros meses del año, cuando todavía los niveles de polen de Gramineae son muy bajos y los de Olea europaea, inexistentes (González Romano et al., 1992). La variación aeropalinológica más homogénea y localizada en el tiempo (PPP de cuatro semanas) es la de Platanus hispanica, como corresponde a un tipo monoespecífico con floración explosiva. En el lado opuesto se encuentran Palmae y Citrus, con curvas polínicas irregulares (producto de la floración escalonada de las numerosas especies que integran dichos tipos).

AGRADECIMIENTOS. Mostramos nuestro agradecimiento al Prof. Santiago Silvestre y al Dr. Felipe García Martín, por el asesoramiento recibido de ambos, que nos ha ayudado a elaborar este trabajo.

\section{BIBLIOGRAFÍA}

ANDRÉS CAMACHO, M.C. -1991- Flora ornamental de Sevilla. Tesis doctoral. Universidad de Sevilla. Sevilla.

CABEZUDO, B., M.M. TRIGO, M. RECIO y F. J. TORO -1994-Contenido polínico de la atmósfera de Málaga: años 1992 y 1993. Acta Bot. Malacitana 19: 137-144.

CAMBON, G.-1981-Relations entre le contenu pollinique de l'atmosphere et le couvert vegetal en Méditérranée occidental à Montpellier
(France), Valencia (Espagne) et Oran (Algerie). Thèse doctorel. Université du Languedoc. Montpellier.

CANDAU, P. \& F.J. GONZÁLEZ MINERO -1992Relationship between vegetation and pollen spectrum in southwest Spain. Israel Journal of Botany 41: 285-295.

CARAMIELLO, R., A. POTENZA, P. MIGLIETTA \& C. SINISCALCO -1992- Re-planning the layout of an inner city park by planting species with a low allergological impact. Aerobiologia 8(1): 133-140.

CEPEDA, J.M. y P. CANDAU -1990- Contribución aeropalinológica al estudio de la influencia de los factores climáticos sobre la floración de Platanus hybrida Brot., Citrus sp. y Olea europaea L.. En BLANCA, G., C. DÍAZ de la GUARDIA, M.C. FERNÁNDEZ, M. GARRIDO, M.I. RODRÍGUEZ-GARCÍA y A.T. ROMERO GARCíA (eds.). Polen, esporas y sus aplicaciones: 329-333. Universidad de Granada. Granada.

COUR, P. -1974- Nouvelles techniques de detection des flux polliniques: étude de la sedimentation des pollens déposés á la surface du sol. Pollen et spores 16: 103-141.

DÍAZ de la GUARDIA, C. y G. BLANCA -1994Flora ornamental de Granada. Polen e incidencia en alergias. Universidad de Granada. Granada.

DONINI, D. \& J.P. SUTRA -1987- Recherches aéropalynologiques à Paris et dans sa banlieue: Nouveaux resultats. Grana 28: 37-44.

ELIAS BONELLS, J.-1983-Plantas y Jardines de Sevilla. Ayuntamiento de Sevilla y Delegación de Parques y Jardines. Sevilla.

FERNÁNDEZ, P., C. DÍAZ de la GUARDIA \& F. VALLE - 1990- Análisis polínico en la atmósfera de Granada, resultados febrero-junio(19891990). An. Asoc. Palinol. Leng. Esp. 5:29-38.

GÁLVEZ, C. y J.L. UBERA- 1985- Nuevas aportaciones al catálogo aeropalinológico de Córdoba: plantas exóticas. An. Asoc. Palinol. Leng. Esp 2: 215-225.

GONZÁLEZ MINERO, F.J. \& P. CANDAU- 1995Análisis del contendio polínico de la atmósfera de Huelva (1989-1992)- Acta Bot. Malacitana 20:71-81.

GONZÁLEZ ROMANO, M.L. \& P. CANDAU 1990-Aportaciones al catálogo aeropalinológico 
de Sevilla. Plantas ornamentales. En BLANCA, G., C. DÍAZ de la GUARDIA, M.C. FERNÁNDEZ, M. GARRIDO, M.I. RODRÍGUEZ-GARCÍA y A.T. ROMERO GARCÍA (eds.). Polen, esporas y sus aplicaciones: 335-346. Universidad de Granada. Granada.

GONZÁLEZ ROMANO, M.L., P. CANDAU \& F.J. GONZÁLEZ MINERO -1992- Pollen calendar of Seville and its relation to allergies. J. Invest. Allergol. Clin. Immunol. 6: 323: 328.

GONZÁLEZ ROMANO, M.L., P. CANDAU y F.J. GONZÁLEZ MINERO- 1993- Estudio aeropalinológico de Sevilla. An. Asoc. Palinol. Leng. Esp. 1993, 6:39-50.

GONZÁLEZ ROMANO, M.L. y CANDAU, P.1994- Aportaciones al catálogo aeropalinológico de Sevilla. Plantas ornamentales II. En La SERNA RAMOS, I. (ed.). Polen y Esporas: contribución a su conocimiento, 169-178. Universidad de La Laguna. Tenerife.

NILSSON, S. \& S. PERSSON -1981- The pollen spectra in the Stockholm region (Sweden), 19731980. Grana 20: 179-182.

RECIO, M., M.M.TRIGO, F.J. TORO y B. CABEZUDO- 1995-Contenido polínico de la atmósfera de Málaga: año 1994. Acta Bot. Malacitana 20: 83-90.

RUIZ de CLAVIJO, E., C. GALÁN, F. INFANTE \& E. DOMÍNGUEZ - 1988- Variations of airborne winter pollen in southern Spain. Allergol. et Immunopathol. 13(3): 175-179.

TRIGO, M.M. e I. GARCÍA -1990- Morfología polínica de plantas ornamentales: Leguminosas. Acta Bot. Malacitana 15:45-68.

TRIGO, M.M. e I. FERNÁNDEZ -1994Contribución al estudio polínico de especies ornamentales con interés alergógeno cultivadas en Málaga: Dicotiledóneas. Acta Bot. Malacitana 19: 145-168.

TRIGO, M.M., I. GARCÍA y B. CABEZUDO 1994- Contribución al atlas palinológico de las especies ornamentales cultivadas en la ciudad de Málaga:Malvales. En La SERNA RAMOS, I. (ed.). Polen y Esporas: contribución a su conocimiento: 105-124. Universidad de La Laguna. Tenerife.
TRIGO, M.M. e I. FERNÁNDEZ -1995Contribución al estudio polínico de especies ornamentales con interés alergógeno cultivadas en Málaga: Monocotiledóneas. Acta Bot. Malacitana 20: 61-70.

VALDÉS, B., DÍEZ, M.J. e I. FERNÁNDEZ - 1987 Atlas polínico de Andalucía Occidental. Instituto de Desarrollo Regional (Universidad de Sevilla).Diputación de Cádiz. Sevilla.

Aceptado para su publicación en Febrero de 1997

Dirección de los autores. Dpto. Biología Vegetal y Ecología. Apdo 874. 41012 Sevilla. 